\title{
Characterization of light contact in head disk interface with dynamic flying height control
}

\author{
Jianfeng Xu • Gang Sheng
}

Received: 11 January 2011/ Accepted: 2 February 2011/Published online: 3 April 2011

(c) The Author(s) 2011. This article is published with open access at Springerlink.com

\begin{abstract}
This paper presents an investigation of the light contact in a head disk interface with dynamic flying height control. The touchdown test is conducted for a dynamic flying height control slider and the response is recorded using AE sensor. The bouncing instability and light contacts are observed during thermal actuated touching down process of the slider. The physics-based simulation is conducted to correlate with the experiments, so as to characterize bouncing instability and the factors affecting bouncing instability. The enhanced spectrogram and HHT approaches are used to extract and characterize the non-stationary characteristics of the weak signal of slider response under light contact. It is found that the light contacts are constituted by a series of intermittent transient impact responses with frequency identical to slider's pitch mode.
\end{abstract}

\section{Introduction}

The head-disk spacing in hard disk drives continues decreasing to sub-10 $\mathrm{nm}$. Under such a low spacing, the short distance forces, such as intermolecular force, begin to

\footnotetext{
J. Xu ( $\square)$

University of California, 9500 Gilman Drive,

La Jolla, San Diego, CA 92093, USA

e-mail: John@talkelab.ucsd.edu

G. Sheng

Department of Mechanical Engineering,

University of Alaska, Fairbanks, AK 99775, USA

e-mail: gsheng@alaska.edu

Present Address:

J. Xu

Western Digital Corporation, 5863 Rue Ferrari,

San Jose, CA 95138, USA
}

play substantial roles. In order to further lower the read/ write transducer to the disk, dynamic flying height (DFH) control sliders have been widely used in air-bearing sliders. Figure 1 shows a schematic view of DFH slider, which employs a heating element integrated in the thin film transducer structure. When power is supplied to this element the slider thermally expands locally in a way that protrudes only a small region around the read/write transducers to move it closer to the disk. Due to the miniature nature of the close-approach region, the destabilizing forces are minimized. DFH control slider has been studied by a number of researchers. Tian et al. (1997) investigated the read back signal disturbance caused by thermally induced flying height fluctuation. They observed that the electrical resistance of the MR element was temperature dependant. Gupta et al. (2001) studied the thermal pole tip protrusion with temperature and current in the write coil. They measured thermal pole tip protrusion using an optical profiler and an atomic force microscope and observed that thermal pole tip protrusion was affected by the material and structure of the read/write head. Pust et al. (2002) simulated thermal pole tip protrusion using three-dimensional finite element analysis and observed that thermal stress in the shields of the head was caused by a mismatch in the coefficient of thermal expansion. They also found that during head operation, the primary heat source was the write coil. Imamura et al. (2002) and Xu et al. (2003) investigated the temperature rise in a slider when flying on a transparent disk using infrared microscopy. Kurita et al. (2005) investigated the flying height reduction of a slider due to thermal pole tip protrusion and found that the protrusion of the pole tip is partially compensated by an increase in the air pressure on the air-bearing surface. Chekanov et al. (2004) studied the effect of thermal pole tip protrusion on the recording performance. They 


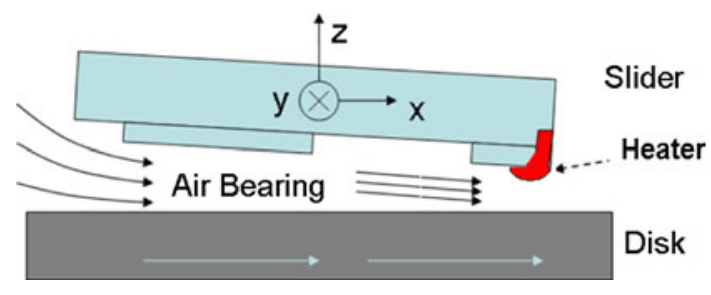

Fig. 1 Schematic view of DFH slider interface

observed that the overwrite degradation was caused by head-disk interactions due to thermal pole tip protrusion and that appropriate selection of disk lubricants could reduce the effect of thermal pole tip protrusion. $\mathrm{Li}$ et al. (2005) simulated the heat transfer between slider and disk surfaces. They showed that the temperature distribution over the air bearing surface was asymmetrical and was related to the air bearing pressure and heat flux distribution. In recent hard disk drives, thermal protrusion of the magnetic read/write element has been used to adjust flying height. Meyer et al. (1999) discussed a heating system in a slider assembly to thermally control the position of the $\mathrm{read} / \mathrm{write}$ element. Dietzel et al. (2000) investigated a method to insert a resistive heater into the substrate of the slider. The expansion of the heater caused a change of the crown of the slider and, consequently, a change in the flying height of the slider. Kurita et al. (2006) investigated an 'active slider' with a nanothermal actuator near the read/ write element. The magnetic spacing of the slider can be controlled in situ during drive operations. Suk et al. (2005) experimentally verified that the magnetic and mechanical spacing could be controlled by thermally actuating the recording transducer. Juang and Bogy (2007) investigated the relationship between air bearing surface design and thermal actuation of a slider. More recently work can be found in (Xu et al. 2009; Hua et al. 2009; Vakis et al. 2009; Shiramatsu et al. 2009; Li et al. 2009; Tani et al. 2009; Ng et al. 2009; Canchi and Bogy 2010; Knigge et al (2006); Li et al. 2010; Canchi and Bogy 2010). Although the above studies have clarified many aspects of current-induced thermal pole tip protrusion, there are still many areas lacking of understanding. For example, the light contact or surfing status of DFH slider has still been debated.

In this paper, we will investigate the dynamics of sliders during light contacts caused by thermal protrusion using experimental and analysis approaches. The touchdown test is conducted for a DFH control slider and the response is recorded by using AE sensors. The bouncing instability and light contacts are observed during thermal actuated touching down process of the slider. The physics-based simulation is conducted to correlate with the bouncing instability and the factors leading to less instability are characterized. The enhanced spectrogram and HHT approaches are used to extract and characterize the weak signal in light contact.

\section{Experimental setup and results}

In this study, the experimental study of the dynamics of thermal actuated slider was conducted using $\mathrm{AE}$ sensor signal. The DFH slider used is an industry-designed femtosized slider. The setup of the experimental system was based on a test stand, which was used for disk rotation, positioning and actuating of DFH slider over disk, and picking up AE signal as shown in Fig. 2. A function waveform generator supplied electric power to the thermal actuator in the DFH slider. The flying height of the sliders was about ten nanometers when sliders were flying over a disk and the electric power was not applied to the thermal actuator.

When a saw-tooth DFH voltage was applied to the thermal actuator in a DFH slider, the interaction between slider and disk occurs when the DFH voltage was high enough, which is shown Fig. 3. The AE signal showed a rise when the DFH voltage reaches to certain threshold. However, when the DFH voltage was further increased, the AE signal dropped quickly to a relatively low level. During the process of the increase in the DFH voltage, the output $\mathrm{AE}$ signal exhibit three distinct regions: region I (without DFH voltage), region II (with threshold DFH voltage) and region III (with saturated DFH voltage), which is consistent with previously observed results (Tang et al. 2007; Zhang et al. 2009; Xu et al. 2009; Hua et al. 2009; Canchi and Bogy 2010).

\section{Analysis and discussion}

To correlate with the experiments, we conducted physicsbased simulation to investigate the bouncing instability in region II in Fig. 3. The simplified model can be derived

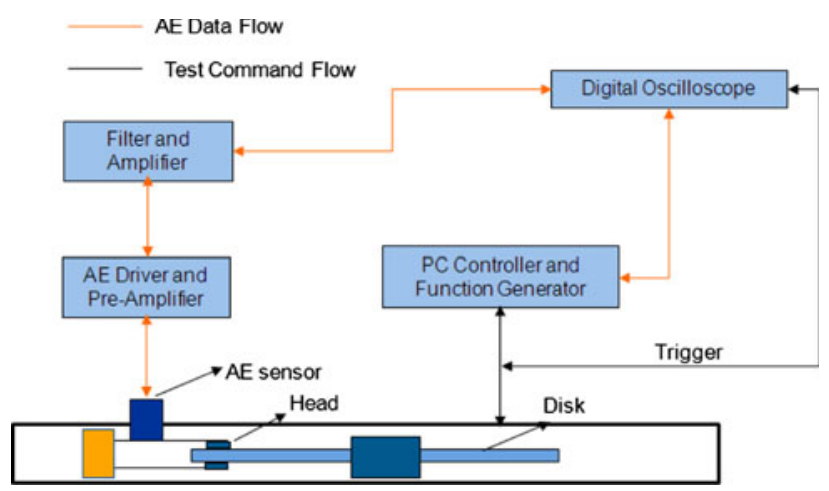

Fig. 2 Schematic of experimental set-up 

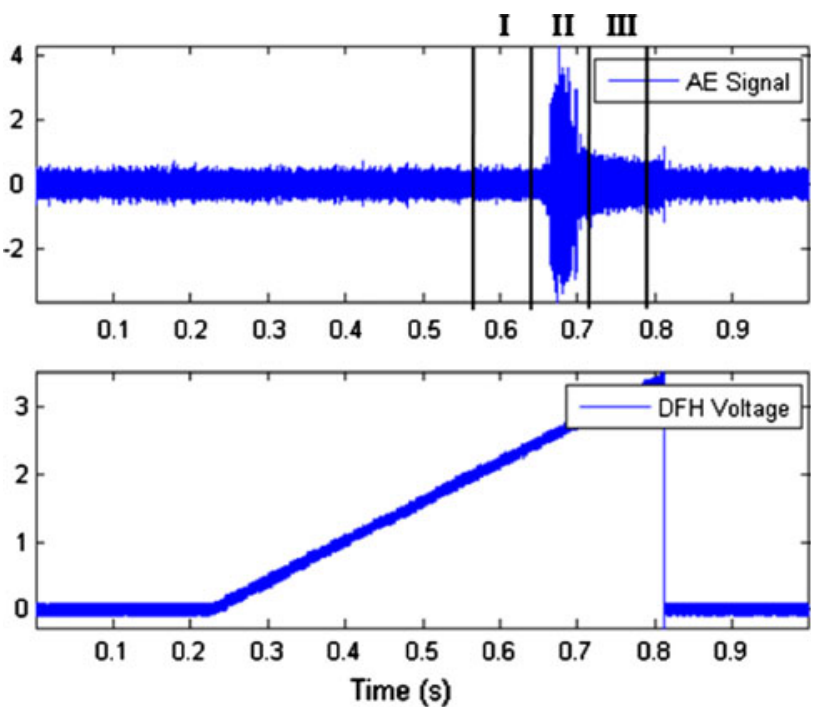

Fig. 3 AE signal of slider response and DFH voltage in touchdown process

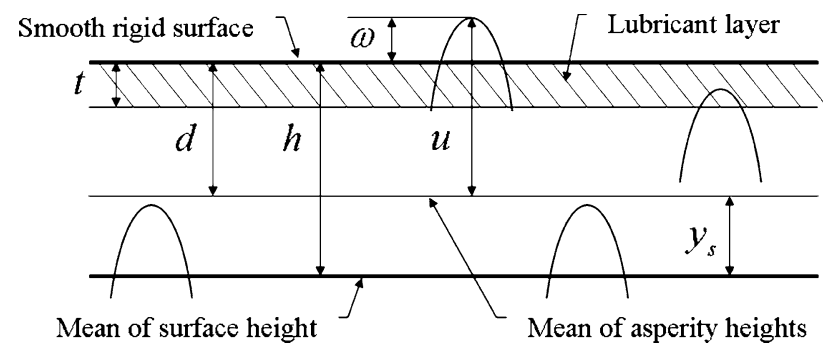

Fig. 4 Illustration of continuum mechanics based contact model. ( $t$ lubricant thickness, $d$ separation, $h$ reference plane to the mean of surface height, $u$ asperity height, $w$ interference height, $\eta$ areal density of asperities, $R$ radius of asperity summit, $A_{n}$ norminal contact area)

from generalized Reynolds equation of air-bearing slider system,

$$
\begin{aligned}
& \frac{\partial}{\partial x}\left(p h^{3} Q_{r} \frac{\partial p}{\partial x}\right)+\frac{\partial}{\partial y}\left(p h^{3} Q_{r} \frac{\partial p}{\partial y}\right) \\
& \quad=6 \mu U_{x} \frac{\partial(p h)}{\partial x}+6 \mu U_{y} \frac{\partial(p h)}{\partial y}+12 \mu \frac{\partial(p h)}{\partial t}
\end{aligned}
$$

where $p(x, y)$ is pressure distribution between a slider and disk surface, $h$ is the flying height distribution, $\mu$ is the viscosity of air, $U x$ and $U y$ are the components of the relative disk velocity respectively, and $Q_{r}$ is the total massflow-rate coefficient. The rigid motion of the slider is described by the following equations,

$$
\begin{aligned}
& m \ddot{z}+F_{s}=f_{z}(t)+\iint\left(p-p_{a}\right) d A \\
& I_{\theta} \ddot{\theta}+M_{s \theta}=f_{\theta}(t)+\iint\left(p-p_{a}\right)\left(x_{g}-x\right) d A \\
& I_{\phi} \ddot{\phi}+M_{s \phi}=f_{\phi}(t)+\iint\left(p-p_{a}\right)\left(y_{g}-y\right) d A
\end{aligned}
$$

where $\mathrm{z}, \theta$ and $\varphi$ are vertical displacement at slider's center, pitch angle and roll angle respectively. $F_{s} M_{s \theta}$ and $M_{s \phi}$ are respectively the force and moments applied by suspension. $f_{z}(t), f_{\theta}(t)$ and $f_{\varphi}(t)$ are external excitation forces, $x_{g}, y_{g}$ are the location of the center of gravity of the slider. $p_{a}$ represents ambient pressure. The above equation can be further simplified as

$[m]\{\ddot{x}\}+[c]\{\dot{x}\}+[k]\{x\}=\{f(t)\}$

where $[m],[k]$, and $[c]$ are the mass, stiffness, and damping matrices, and $\{x\}$ is the vibration displacement vector of the slider. In previous work, a variety of nonlinearities such as contact, nonlinear air-bearing, friction, lubricant adhesion and intermolecular forces were explored theoretically or numerically by incorporating them into Eq. (2) or Eq. (3). In present work, a modified SBL (Kogut and Etsion, 2004; Suh and Polycarpou 2005) model is used. An illustration of continuum mechanics based contact model is shown in Fig. 4. Some parameters used in the formulas is normalized with the standard derivation of surface height $\sigma$.

The adhesive force $F_{s}$ is given by:

$$
\begin{aligned}
F_{3}= & 2 \pi \eta R A_{x} \Delta y\left\{\int_{-\infty}^{a^{+}-t^{+}} J_{n c}+\int_{a^{+}-t^{+}}^{a^{+}} J_{I c}+0.98 \int_{a^{+}}^{a^{+}+w_{0}^{+}} J_{-0.29}^{0.289}\right. \\
& \left.+0.79 \int_{a^{+}+w_{0}^{+}}^{a^{+}} J_{-0321}^{0356}+1.19 \int_{a^{+}+6 w_{0}^{+}}^{a^{+}+110 w_{0}^{+}} J_{-0332}^{0.093}\right\}
\end{aligned}
$$

where the integral on $J_{n c}$ corresponding to contribution of non-contacting asperities, the integral on $J_{l c}$ corresponding to contribution of lubricant contacting asperities and the integrals on $J_{c}^{b}$ corresponding to contribution of contacting asperities.

The contact force $P$ is given by:

$$
\begin{aligned}
P= & \frac{2}{3} \pi \beta K A_{n} H \omega_{c}^{+}\left\{\int_{a^{+}-t^{+}}^{a^{+}+w_{0}^{+}} I^{1.5}+1.03 \int_{a^{+}+w_{0}^{+}}^{a^{+}+6 w_{0}^{+}} I^{14.25}\right. \\
& \left.+1.4 \int_{a^{+}+6 w_{0}^{+}}^{a^{+}+110 w_{0}^{+}} I^{1263}+\frac{3}{K} \int_{a^{+}+110 w_{0}^{+}}^{+\infty} I\right\}
\end{aligned}
$$

where $I b$ is the general form of the integrand:

$I_{b}=\left(\frac{u^{*}-d^{*}}{\omega_{c}^{*}}\right) \phi^{*}\left(u^{*}\right) d u^{*}$

The model provides the calculation of maximum static friction force $Q_{\max }$. We give an estimated ratio of $2 / 3$ for transfer the static friction force to dynamic friction force. 
Table 1 Parameters for material properties used in contact force calculation

\begin{tabular}{llll}
\hline Parameters & Symbol & Unit & Value \\
\hline Young's moduli & $E_{\text {disk }}, E_{\text {slider }}$ & $\mathrm{GPa}$ & 100,450 \\
Poisson's ratio & $v_{\text {disk }}, v_{\text {slider }}$ & & $0.30,0.30$ \\
Hardness of the softer material (disk) & $H$ & $\mathrm{GPa}$ & 3.6 \\
Mobile lubricant thickness & $t$ & $\mathrm{~nm}$ & 0.5 \\
Energy of adhesion & $\Delta \gamma$ & $\mathrm{N} / \mathrm{m}$ & 0.09 \\
Contact area & $A$ & $\mu \mathrm{m}^{2}$ & 1,000 \\
Standard deviation of surface height & $\sigma$ & $\mathrm{nm}$ & 0.50 \\
Standard deviation of combined asperity height & $\sigma_{s}$ & $\mathrm{~nm}$ & 0.40 \\
Intermolecular distrance & $\varepsilon$ & $\mathrm{nm}$ & $0.3 \sim 0.5$ \\
Area density of asperities & $\eta$ & $\mu \mathrm{m}^{-2}$ & 10.0 \\
Radius of asperity summit & $R$ & $\mu \mathrm{m}$ & 7.0 \\
Gap between mean asperity height and mean surface height & $y_{s}$ & $\mathrm{~nm}$ & 0.70 \\
\hline
\end{tabular}

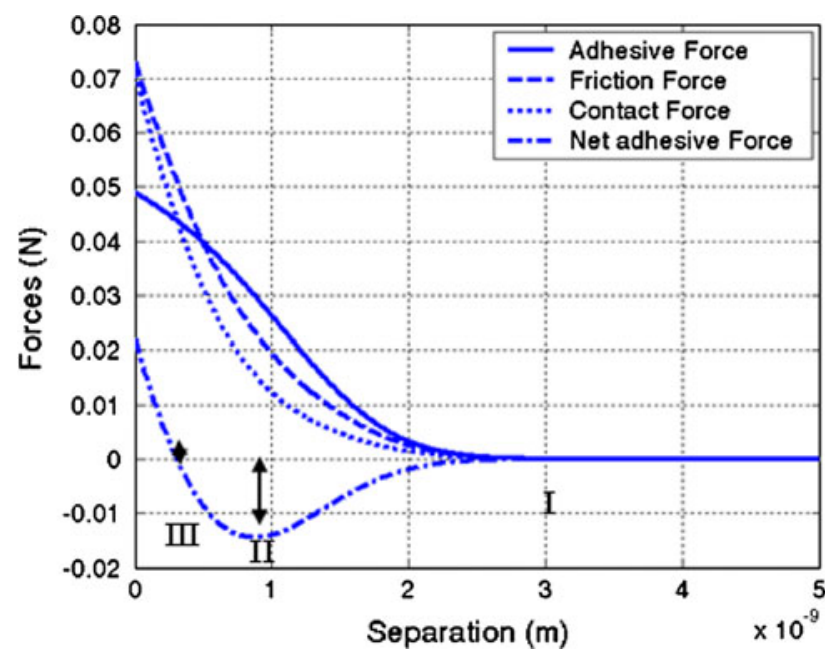

Fig. 5 Force calculated using the modified SBL model

$$
\begin{aligned}
Q_{\max } & =\frac{2}{3} \pi \beta K A_{n} H \omega_{c}^{*}\left\{0.52 \int_{a^{+}+t^{+}}^{a^{+}+w_{0}^{+}} I^{0982}\right. \\
+ & \left.\int_{a^{+}+w_{0}^{+}}^{a^{+}+6 w_{0}^{+}}\left(-0.01 I^{4.425}+0.09 I^{3.425}-0.85 I^{1.425}\right)\right\}
\end{aligned}
$$

The parameters used for calculation of forces have been listed on Table 1.

Referencing Eqs. 4, 5 and 7, forces calculated using the parameters shown in Table 1 incorporated with modified SBL model is shown in Fig. 5.

The net adhesive force (derived from the adhesive force deducted by the contact force) and the friction force are used for estimate slider motion. From Fig. 5 it could be found that there is a negative region II for net adhesive

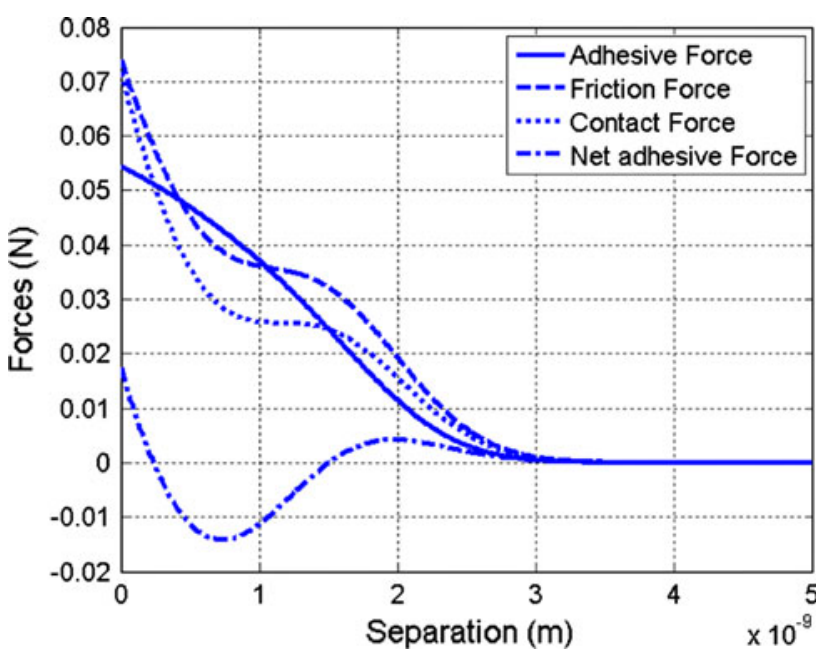

Fig. 6 Force calculated with lubricant thickness increased by fifty percent

force, which is corresponding to the measured bouncing instability region II in Fig. 3.

When the spacing was further lower to region III shown on Fig. 5, the adhesive force and the contact force went into a balanced condition. The level of the net adhesive force was reduced so as that air bearing sliders could achieve a relative stabilized condition, or so-called light contact condition. At this condition, the air bearing slider vibrations would be limited, corresponding to region III shown in Fig. 3.

To achieve a quickly stabilized contact condition, one could maximize the net adhesive force region. However, for utilizing the region III as a stable recording condition, the net adhesive force region should be minimized.

To further explore the effect of interfacial parameters on the net adhesive force, the lubricant thickness was increased by fifty percent, the simulation results are shown in Fig. 6. From Figs. 5 and 6 it can be found that after the 


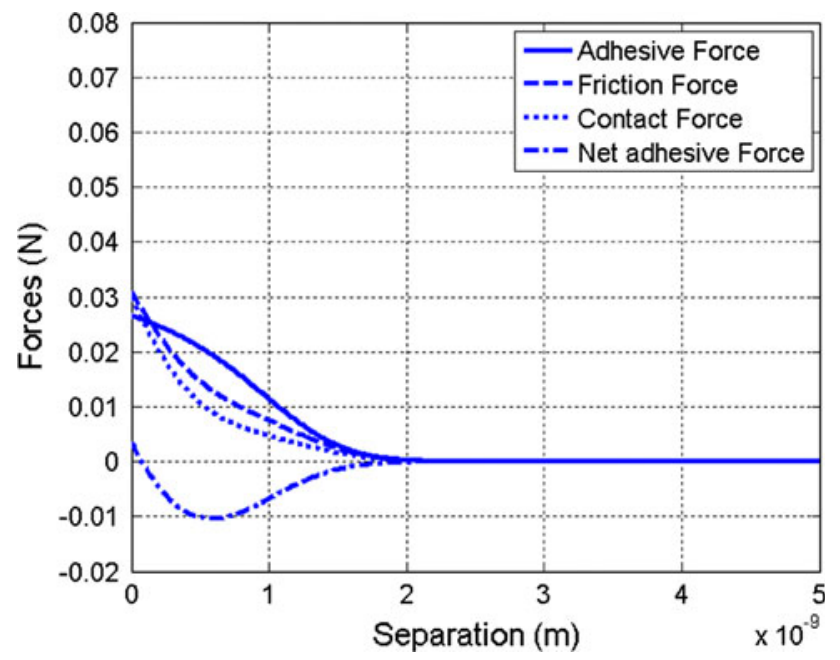

Fig. 7 Force calculated with surface roughness decreased by twenty five percent

lubricant thickness increases, the net adhesive force increases first before switch to negative region similar to original case, whereas friction force increases.

In the continuing study the surface roughness was decreased by twenty five percent, the simulation results are shown in Fig. 7. From Fig. 5 and 7 one can find that after surface roughness decreased, the amplitude of the net adhesive force decreases about thirty percent compared with original case at the negative region, whereas friction force also decreases.

In the next we look into the light contact in the region III in Fig. 3. The light contact or lubricant surfing has been widely discussed (Vakis et al. 2009; Ng et al. 2009; Canchi and Bogy 2010; Li et al. 2010). In order to extract information from the weak signal in the region III in Fig. 3, we apply advanced time-frequency analysis, which include enhance spectrogram and empirical mode decomposition
(EMD) (Huang and Attoh-Okine 2005). Figure 8a shows the enhanced spectrogram of the data from region III in Fig. 3. As a comparison, Fig. $8 \mathrm{~b}$ shows the enhanced spectrogram of the data from region I in Fig. 3.

It can be seen that the slider vibrations in region III exhibit a non-stationary feature, which has fixed natural frequencies 150 and $204 \mathrm{kHz}$ respectively. These two frequencies correspond to slider air-bearing's pitch modes. To further explore the response pattern, the dominant component of $204 \mathrm{kHz}$ is extracted by using EMD approach that serves as efficient adaptive filter that is suitable for non-stationary, nonlinear signal. Figure 9 shows the dominant component $(204 \mathrm{kHz})$ of data in region III in Fig. 3 obtained by using EMD. We can see that in region III, the slider dominant response consists of a series of intermittent rise and decay waves with identical frequency $(204 \mathrm{kHz})$. This suggests that slider runs into objects blocking its path, which could be due to lubricant asperity. This effect causes slider transient free-decay response. This observation offers some insight to the light contact or lubricant surfing.

In principle, the short-range forces and bouncing effect should cause complex nonlinear slider vibration such as harmonics, but the light contact oscillation exhibits the simple linear patterns as shown in Figs. 8 and 9, which actually represents free-decay response of slider air-bearing mode under light, intermittent and consecutive impacts.

\section{Conclusion}

This paper presents an investigation of the light contact in a head disk interface with dynamic flying height control. The touchdown test is conducted for a dynamic flying height control slider and the response is recorded using AE sensor. The bouncing instability and light contacts are observed during thermal actuated touching down process of slider.
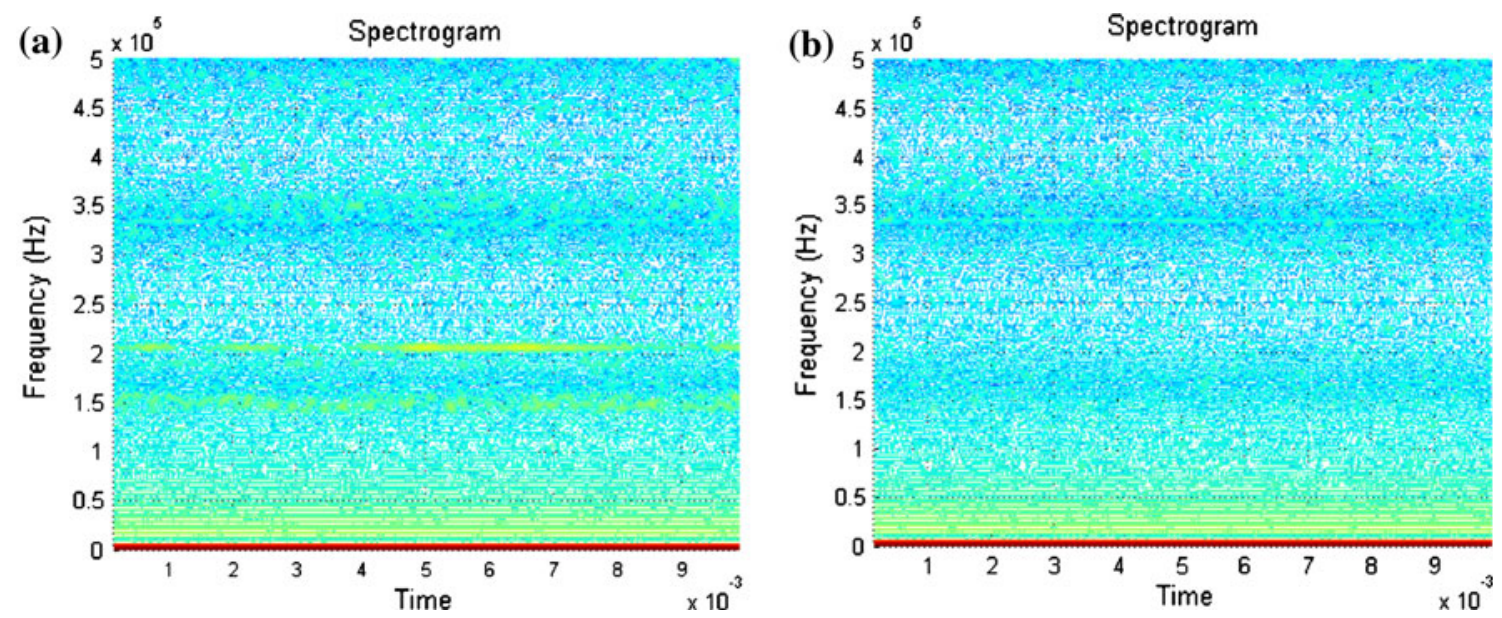

Fig. 8 Enhanced spectrogram: a data from region III in Fig. 3 and $\mathbf{b}$ data from region I in Fig. 3 


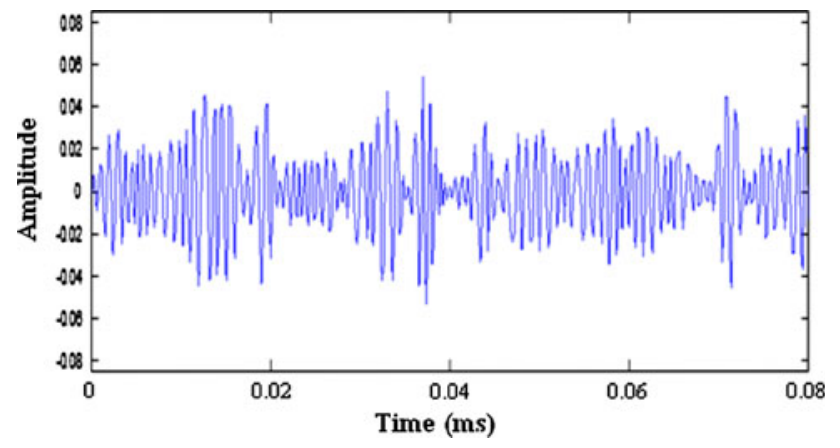

Fig. 9 The dominant component $(204 \mathrm{kHz})$ of data in region III in Fig. 3 by using EMD

The physics-based simulation is conducted to correlate with the experiments, so as to characterize bouncing instability and the factors affecting bouncing instability. The enhanced spectrogram and HHT approaches are used to extract and characterize the non-stationary characteristics of the weak signal of slider response under light contact. The light contacts are constituted by a series of intermittent transient impact responses with frequency identical to slider's pitch mode.

Acknowledgments Part of this paper was derived from the work when Jianfeng Xu was doing his Ph.D. study under Professor Frank E. Talke at University of California, San Diego.

Open Access This article is distributed under the terms of the Creative Commons Attribution Noncommercial License which permits any noncommercial use, distribution, and reproduction in any medium, provided the original author(s) and source are credited.

\section{References}

Canchi SV, Bogy DB (2010) Slider dynamics in the lubricant-contact regime. IEEE Trans Magn 46(3):764-769

Chekanov A, Weng R, Li F (2004) Pole-tip protrusion effect on high data rate recording performance. IEEE Trans Magn 40:2591-2593

Dietzel A, Berger R, Machtle P, Despont M, Haberle W, Stutz R, Binning GK, Vettiger P (2000) In situ slider-to-disk spacing on a nanometer scale controlled by microheater-induced slider deformation. Sens Actuators A Phys 100:123-130

Gupta BK, Young K, Chilamakuri SK, Menon AK (2001) On the thermal behavior of giant magnetoresistance heads. ASME J Tribol 123:380-387

Hua W, Liu B, Yu SK, Zhou WD (2009) Nanoscale roughness contact in a slider-disk interface. Nanotechnology 20:285710

Huang NE, Attoh-Okine NO (2005) The Hilbert-Huang transform in engineering. CRC Press, Boca Raton

Imamura T, Yamagishi M, Nishida S (2002) In situ measurement of temperature distribution of air bearing surface using thermography. IEEE Trans Magn 38:2147-2149
Juang JY, Bogy DB (2007) Air-bearing effects on actuated thermal pole-tip protrusion for hard disk drives. ASME J Tribol 129:570-578

Knigge B, Suthar S, Baumgart P (2006) Friction, heat, and slider dynamics during thermal protrusion touchdown. IEEE Trans Magn 42(10):1-3

Kogut L, Etsion I (2004) A static friction model for elastic-plastic contacting rough surfaces. ASME J Tribol 126:34-40

Kurita M, Xu JG, Tokuyama M (2005) Flying-height reduction of magnetic head slider due to thermal protrusion. IEEE Trans Magn 41:3007-3009

Kurita M, Shiramatsu T, Miyake K, Kato A, Soga M, Tanaka H, Saegusa S, Suk M (2006) Active flying-height control slider using MEMS thermal actuator. Microsyst Technol 12:369-375

Li H, Liu B, Chong TC (2005) Numerical simulation of slider temperature rise caused by read/write current. J Appl Phys 97:306-308

Li N, Zheng LS, Meng YG, Bogy DB (2009) Experimental study of head-disk interface flyability and durability at sub-1-nm clearance. IEEE Trans Magn 45(10):3624-3627

Li JH, Xu JG, Aoki Y (2010) Simulation on contact between the droplet and the slider at head-disk interface based on waterhammer pressure model. Microsyst Technol 16:57-65

Meyer D, Kupinski PE, Liu JC (1999) Slider with responsive transducer positioning. US Patent 5991113

Ng L, Zhang MS, Liu B, Ma YS (2009) Slider-disk contact characterization using a thermal fly-height control slider. IEEE Trans Magn 45(11):5026-5029

Pust L, Rea CJT, Gangopadhyay S (2002) Thermomechanical head performance. IEEE Trans Magn 38:101-106

Shiramatsu T, Atsumi T, Kurita M, Shimizu Y, Tanaka H (2009) Dynamically controlled thermal flying-height control slider. IEEE Trans Magn 44(11):3695-3697

Suh AY, Polycarpou AA (2005) Adhesive contact modeling for sub5 -nm ultralow flying magnetic storage head-disk interfaces including roughness effects. J Appl Phys 97:104328

Suk M, Miyake K, Kurita M, Tanaka H, Saegusa S, Robertson N (2005) Verification of thermally induced nanometer actuation of magnetic recording transducer to overcome mechanical and magnetic spacing challenges. IEEE Trans Magn 41:4350-4352

Tang Y, Hong S-Y, Kim N-Y, Che X (2007) Overview of fly height control applications in perpendicular magnetic recording. IEEE Trans Magn 43(2):709-711

Tani H, Kanda M, Kubota M, and Tagawa N (2009) Study of sliderdefect interaction at ultralow flying height by dynamic flying height control. J Appl Phys 105:07B703

Tian H, Cheung C-Y, Wang P-K (1997) Non-contact induced thermal disturbance of MR head signals. IEEE Trans Magn 33:3130 3132

Vakis AI, Lee SC, Polycarpou AA (2009) Dynamic head-disk interface instabilities with friction for light contact (surfing) recording. IEEE Trans Magn 45(11):4966-4971

Xu J, Tokuyama M, Kurita M, Maruyama Y (2003) High resolution measurement of temperature distribution of head coil and airbearing surface. IEEE Trans Magn 39:2411-2413

Xu JF, Kiely JD, Hsia YT, Talke FE (2009) Effect of thermal pole tip protrusion and disk roughness on slider disk contacts. Microsyst Technol 15:687-693 